\title{
SUR CERTAINES RELATIONS ENTRE LES INTÉGRALES TRAJECTORIELLES ET L'OPÉRATEUR DE TRANSLATION ET SON DUAL DANS L'ESPACE DE POISSON CANONIQUE
}

\author{
Jorge A. León, Josep L. Solé et Josep Vives
}
Abstract
We study the relationship between the translation operator, its dual and the pathwise integral on the Poisson space with weak conditions on the processes.

\section{Introduction}

Le calcul stochastique associé au processus de Poisson a interessé de nombreux auteurs. Se placer dans l'espace canonique de Poisson permet de faire des démonstrations trajectorielles directes (voir $[\mathbf{N e}],[\mathbf{N}-\mathbf{V - 2}]$ ). Nualart et Vives [N-V-1], [N-V-2] ont introduit l'opérateur de translation $\Psi$ et son dual, l'opérateur $\Phi$, qui ont de très bonnes propriétés, mais ils travaillent dans un cadre $L^{2}$ qui n'est peut-être pas complètement approprié.

Dans la section 2 nous rappellons tous ces concepts, et présentons quatre lemmes sur les opérateurs $\Phi$ et $\Psi$ dont nous aurons besoin plus tard.

La section 3 est consacrée à la relation entre ces opérateurs et les intégrales trajectorielles dans un cadre général, mais pour l'établir on a besoin de fixer une version concrète du processus et des conditions d'intégrabilité. La formule à laquelle nous arrivons généralise dans le cas Poisson (c'est à dire avec $\phi=1$, où $\phi$ est le processus qui apparaît dans l'équation de structure), une expression bien connue pour les probabilistes quantiques (Prop. 18 de Biane $[\mathbf{B}]$ ).

The authors have been supported by DGICYT, grant PB93-0052, PB96-1182, and CIRIT, grant 97-SGR00144. Also, the first author has been partially supported by CONACyT. 
En outre, cette formule et la relation entre l'intégrale $\delta$, définie à partir de la décomposition chaotique, et l'opérateur $\Phi$ dans $L^{2}[\mathbf{N}-\mathbf{V}-2]$, nous permettent de préciser et de prouver aisement un théorème de NualartVives $[\mathbf{N}-\mathbf{V - 1}]$, qui lie cette intégrale $\delta$ avec l'intégrale trajectorielle, publié dans le Séminaire de Probabilités XXIV.

Dans la section 4 on considère les intégrales progressive et rétrograde dans le cas Poissonnien en un sens trajectoriel, et on donne un point de vue différent sur la formule trouvée à la section précédente, pour des processus càdlàg avec certaines hypothèses supplémentaires.

Notre intérêt pour les intégrales rétrogrades et progressives est motivé par le bien connu problème des équations intégrales au sens trajectoriel conduites par un processus de Poisson, qui n'ont pas de solution si l'intégrale est rétrograde, et qui en ont si elle est progressive.

\section{Le calcul stochastique sur l'espace de Poisson canonique}

Considérons l'intervalle $T=[0,1]$ avec sa tribu borelienne $\mathcal{B}$ et la mesure de Lebesgue $\lambda$.

On définit $\Omega=\bigcup_{n=0}^{\infty}[0,1]^{n}$, espace de toutes les suites finies de points de $[0,1]$, avec $[0,1]^{0}:=\{e\}$ où $e$ est un point arbitraire.

Soit $\mathcal{F}$ la tribu

$$
\left\{G \subseteq \Omega: G \cap[0,1]^{n} \in \mathcal{B}^{\otimes n}, \text { pour tout } n \geq 1\right\} .
$$

Finalement, soit $\lambda^{n}$ la mesure produit sur $[0,1]^{n}$, pour $n \geq 1$ et $\lambda^{0}=\delta_{e}$.

$\operatorname{Sur}(\Omega, \mathcal{F})$ on peut définir la mesure de probabilité suivante:

$$
P(G)=\sum_{n=0}^{\infty} \frac{e^{-1}}{n !} \lambda^{n}\left(G \cap[0,1]^{n}\right) .
$$

Soit $M_{p}([0,1])$ l'espace des mesures ponctuelles finies sur $[0,1]$, et $\mathcal{M}_{p}([0,1])$ la tribu engendrée par les applications $m \rightarrow m(F)$, avec $F \in \mathcal{B}$ et $m \in M_{p}([0,1])$.

Il est bien connu (voir $[\mathbf{N e}],[\mathbf{N}-\mathbf{V}-\mathbf{1}]$ ) que l'application

$$
N: \Omega \longrightarrow M_{p}([0,1])
$$

définie par

$$
N(\omega):= \begin{cases}0, & \text { si } \omega=e \\ \sum_{i=1}^{n} \delta_{s_{i}}, & \text { si } \omega=\left(s_{1}, \ldots, s_{n}\right)\end{cases}
$$

est un processus de Poisson qui a les propriétés suivantes: 
(1) Pour chaque borélien $B$ de $[0,1]$, la variable aléatoire $N(B)$ définie par $N(B)(\omega)=N(\omega)(B)$ a une distribution de Poisson de moyenne $\lambda(B)$.

(2) Les variables aléatoires $N\left(B_{1}\right), \ldots, N\left(B_{n}\right)$ sont indépendantes si $B_{1}, \ldots, B_{n}$ sont des boréliens disjoints.

De plus, si $\mathcal{B}_{S}^{\otimes n}$ est la sous-tribu des boréliens symétriques de $[0,1]^{n}$, on peut définir la tribu $\mathcal{F}_{S}$, associée à $\Omega$, des $G \in \mathcal{F}$ tels que $G \cap[0,1]^{n} \in$ $\mathcal{B}_{S}^{\otimes n}$ pour $n \geq 1$. On a aussi que $N^{-1}\left(\mathcal{M}_{p}\right)=\mathcal{F}_{S}$.

Dans la suite on travaillera sur $\left(\Omega, \mathcal{F}_{S}, P\right)$, c'est à dire sur l'espace de Poisson canonique sur $([0,1], \mathcal{B}, \lambda)$.

Denotons par $\tilde{N}=\left\{N_{s}-s, s \in[0,1]\right\}$, le processus de Poisson compensé, où $N_{s}=N([0, s])$.

Remarque 2.1. Dans l'espace de Poisson canonique,

$$
\Omega^{*}=\bigcup_{n=0}^{\infty}\left\{\left(s_{1}, \ldots, s_{n}\right): 0 \neq s_{1} \neq \cdots \neq s_{n} \neq 1\right\} \cup\{e\}
$$

est un ensemble symétrique de $\mathcal{F}_{S}$ qui a probabilité 1 . Donc on ne considérera que des suites de ce type.

Notons que si $F$ est une variable aléatoire $\mathcal{F}_{S}$-mesurable, il suffit de définir $F(e)$ et $F\left(s_{1}, \ldots, s_{n}\right)$ pour $0<s_{1}<\cdots<s_{n}<1$ et puis, comme conséquence de la mesurabilité on a

$$
F\left(s_{\sigma(1)}, \ldots, s_{\sigma(n)}\right)=F\left(s_{1}, \ldots, s_{n}\right)
$$

pour toute permutation $\sigma$.

Remarque 2.2. La suite $\left\{s_{1}, \ldots, s_{n}\right\}$ avec $0<s_{1}<\cdots<s_{n}<1$ peut s'interpréter comme la suite des temps de saut du processus de Poisson et $e$ représente la trajectoire sans saut.

Suivant $[\mathbf{N}-\mathbf{V}-\mathbf{1}]$ nous allons définir un couple d'opérateurs qui permettent de construire un calcul dans ce contexte.

Si $\omega \in \Omega$, nous definissons

$$
\omega+\delta_{t}= \begin{cases}\left(s_{1}, \ldots, s_{n}, t\right), & \text { si } \omega=\left(s_{1}, \ldots, s_{n}\right) \\ (t), & \text { si } \omega=e .\end{cases}
$$

Soit $F$ une variable aléatoire $\mathcal{F}_{S}$-mesurable. On définit l'opérateur $\Psi$ comme $\Psi_{t} F(\omega)=F\left(\omega+\delta_{t}\right)-F(\omega)$, p.s.

Notons que l'opérateur $\Psi$ est bien défini par le lemme suivant [L-R-T].

Lemme 2.3. Soit $A$ un ensemble mesurable de probabilité 1. Alors $(A-\{e\}) \times\{t\} \subset A$ pour presque tout $\omega \in(A-\{e\})$ pour presque tout $t \in T$. 
Soit par ailleurs $u=\left\{u_{s}, s \in[0,1]\right\}$ un processus stochastique mesurable avec trajectoires dans $L^{1}([0,1])$, p.s.

On définit

$$
(\Phi(u))\left(s_{1}, \ldots, s_{n}\right)=\sum_{j=1}^{n} u_{s_{j}}\left(s_{1}, \ldots, \hat{s}_{j}, \ldots, s_{n}\right)-\int_{0}^{1} u_{s}\left(s_{1}, \ldots, s_{n}\right) d s,
$$

pour $n \geq 2$, où le chapeau veut dire que l'on omet $s_{j}$, et

$$
\begin{aligned}
& (\Phi(u))\left(s_{1}\right)=u_{s_{1}}(e)-\int_{0}^{1} u_{s}\left(s_{1}\right) d s \\
& (\Phi(u))(e)=-\int_{0}^{1} u_{s}(e) d s .
\end{aligned}
$$

Une adaptation du lemme précédent où l'on enlève $t$ au lieu de l'ajouter nous montre que l'opérateur $\Phi$ est également bien défini.

Alors, $\Psi: L^{0}(\Omega) \rightarrow L^{0}(\Omega \times[0,1])$ et $\Phi: L^{0}\left(\Omega, L^{1}([0,1])\right) \rightarrow L^{0}(\Omega)$ sont des opérateurs duaux. $\mathrm{Si}$

$$
E \int_{0}^{1}\left|F \cdot u_{t}\right| d t<\infty
$$

on a $F \cdot \Phi(u) \in L^{1}(\Omega)$ si et seulement si

$$
E \int_{0}^{1}\left|\Psi_{t} F \cdot u_{t}\right| d t<\infty
$$

et dans ce cas

(voir $[\mathbf{N}-\mathbf{V}-\mathbf{1}])$.

$$
E[F \cdot \Phi(u)]=E \int_{0}^{1} \Psi_{t} F \cdot u_{t} d t
$$

Il est connu aussi que $\Phi$ coïncide avec l'intégrale trajectorielle sur les processus prévisibles, par rapport au processus de Poisson compensé $\tilde{N}_{t}=N_{t}-t$.

Nous présentons dans la suite quelques résultats dont nous aurons besoin dans les sections suivantes. Le premier et le second lemme se trouvent dans León, Ruiz et Tudor (voir $[\mathbf{L}-\mathbf{R}-\mathbf{T}]$ ). Le premier est un théorème de Fubini anticipatif:

Lemme 2.4. Soit $u$ un processus mesurable dans $L^{1}\left([0,1]^{2}\right) \omega$-p.s. tel que $\Phi(u(s, \cdot)) \in L^{1}([0,1])$ w-p.s. Alors

$$
\int_{0}^{1} \Phi(u(s, \cdot)) d s=\Phi\left(\int_{0}^{1} u(s, \cdot) d s\right), \quad \omega-p . s .
$$

Le second lemme est une conséquence de la définition des opérateurs $\Phi$ et $\Psi$. 
Lemme 2.5. Soit $G$ une variable aléatoire et $u$ un processus tels que $u$ et $\Psi G \cdot u=\left\{\Psi_{s} G \cdot u_{s}, s \in[0,1]\right\}$ sont deux processus mesurables à trajectoires dans $L^{1}([0,1])$. Alors

$$
\Phi(G \cdot u)=G \cdot \Phi(u)-\Phi(\Psi G \cdot u)-\int_{0}^{1} u_{s} \Psi_{s} G d s, \quad \omega-p . s .
$$

Le lemme suivant nous sera utile dans la dernière section.

Lemme 2.6. Soient $\left\{u, u^{n} ; n \in N\right\}$ une famille de processus mesurables à trajectoires dans $L^{1}([0,1])$, tels que

$$
u_{t}^{n}(w) \longrightarrow u_{t}(w)
$$

ponctuellement et dans $L^{1}([0,1])$, w-p.s. Alors on a

$$
\Phi\left(u^{n}\right) \stackrel{n \rightarrow \infty}{\longrightarrow} \Phi(u), \quad \omega-p . s .
$$

Preuve: Etant donné $\omega=\left(s_{1}, \ldots, s_{n}\right)$,

$$
\begin{array}{r}
\left|\Phi(u)-\Phi\left(u^{n}\right)\right| \leq \sum_{j=1}^{n}\left|u_{s_{j}}\left(s_{1}, \ldots, \hat{s}_{j}, \ldots, s_{n}\right)-u_{s_{j}}^{n}\left(s_{1}, \ldots, \hat{s}_{j}, \ldots, s_{n}\right)\right| \\
+\int_{0}^{1}\left|u_{t}-u_{t}^{n}\right| d t,
\end{array}
$$

et les hypothèses impliquent le résultat souhaité.

Les cas $\omega=e$ et $\omega \in T$ se traitent de manière analogue.

\section{La relation entre l'opérateur $\Phi$ et l'intégrale trajectorielle}

Proposition 3.1. Soit $u=\left\{u_{t}, t \in[0,1]\right\}$ une version fixée d'un processus stochastique mesurable défini sur $\Omega \times[0,1]$, $\omega$-p.s., et pour tout $t \in[0,1]$. Supposons que les processus $u$ et $\Psi u=\left\{\Psi_{s} u_{s}, s \in[0,1]\right\}$ ont des trajectoires dans $L^{1}([0,1]), \omega$-p.s. Alors pour presque tout $\omega \in \Omega$ on $a$

$$
\int_{0}^{1} u_{s} d \tilde{N}_{s}=\Phi(u)+\Phi(\Psi u)+\int_{0}^{1} \Psi_{s} u_{s} d s
$$

ou $\int_{0}^{1} u_{s} d \tilde{N}_{s}$ est l'intégrale trajectorielle. 
Preuve: Si $\omega=\left(s_{1}, \ldots, s_{n}\right)$ avec $n>1$,

$$
\begin{aligned}
\int_{0}^{1} u_{s} d \tilde{N}_{s}-\Phi(u)= & \sum_{j=1}^{n}\left\{u_{s_{j}}\left(s_{1}, \ldots, s_{n}\right)-u_{s_{j}}\left(s_{1}, \ldots, \hat{s}_{j}, \ldots, s_{n}\right)\right\} \\
= & \sum_{j=1}^{n} \Psi_{s_{j}} u_{s_{j}}\left(s_{1}, \ldots, \hat{s}_{j}, \ldots, s_{n}\right)=\Phi(\Psi u) \\
& +\int_{0}^{1} \Psi_{s} u_{s} d s
\end{aligned}
$$

et on procède de manière analogue pour $\omega=e$ et $\omega \in T$.

Remarque 3.2. Si $u$ a des limites à gauche qui satisfont les conditions de la Proposition 3.1 on a

$$
\int_{0}^{1} u_{s-} d \tilde{N}_{s}=\Phi\left(u_{-}\right)+\Phi\left(\Psi u_{-}\right)+\int_{0}^{1} \Psi_{s} u_{s-} d s .
$$

Remarque 3.3. Notons que si $u$ est un processus prévisible on a $u_{t}(e)=u_{t}(t)$ et $u_{s_{j}}\left(s_{1}, \ldots, s_{n}\right)=u_{s_{j}}\left(s_{1}, \ldots, \hat{s}_{j}, \ldots, s_{n}\right)$ (voir Nualart et Vives $[\mathbf{N}-\mathbf{V}-\mathbf{1}])$. Alors si $u$ satisfait les conditions de 3.1, cette proposition implique que $\int_{0}^{1} u_{s} d \tilde{N}_{s}=\Phi(u)$.

Remarque 3.4. Notons que pour presque tout $\omega$ on doit prendre une version concrète du processus $u$ sur tout $t \in[0,1]$ pour donner un sens à $\Psi_{s} u_{s}$.

Par ailleurs, la condition d'intégrabilité des processus $u$ et $\Psi u$ assure que tous les termes qui apparaissent dans la preuve existent.

Remarque 3.5. Dans le domaine des probabilités quantiques, la formule de la Proposition 3.1 était déjà connue quand $u=F K_{s}$, où $K$ est un processus prévisible borné et $F$ une variable exponentielle (voir [B, Proposition 18]). Biane donne cette formule dans le cadre des martingales normales avec $\phi$ (processus qui apparait dans l'équation de structure) plus général qu'une constante. Nous nous sommes placés dans le cas Poissonnien $(\phi=1)$, mais notre formule est valable pour une classe plus générale de processus $u$. Une preuve non quantique de la formule de Biane ainsi que certaines conséquences intéressantes peuvent se trouver dans $[\mathbf{P}-\mathbf{S}-\mathbf{V}]$.

Comme application de la Proposition 3.1 on peut établir la relation entre l'intégrale $\delta$ et l'intégrale trajectorielle dans le cas Poissonnien. Ceci corrige un résultat de Nualart et Vives ([N-V-1, Théorème 7.3]) 
publié dans le Séminaire de Probabilités XXIV, qui est placé dans un cadre $L^{2}$ et où il y a des expressions telles que $D_{s} u_{s}$ et $D_{s} D_{s} u_{s}$, où $D$ est l'opérateur d'annihilation, qui n'ont pas de sens dans ce cadre.

Toujours dans un cadre $L^{2}$, Nualart et Vives $[\mathbf{N}-\mathbf{V - 2}]$ donnent la proposition suivante qui lie l'intégrale $\delta$, définie à partir de la décomposition chaotique, avec l'opérateur $\Phi$.

Proposition 3.6. Soit $u=\left\{u_{t}, t \in[0,1]\right\}$ un processus stochastique de $L^{2}(\Omega \times[0,1])$. Alors $\Phi(u) \in L^{2}(\Omega)$ si et seulement si u est dans le domaine de $\delta$, et dans ce cas $\delta(u)=\Phi(u)$.

Considérons donc un processus $u$ satisfaisant les conditions de la Proposition 3.1 et supposons aussi qu'il soit dans $L^{2}(\Omega \times[0,1])$. Si $\Phi(u) \in L^{2}(\Omega)$, la Proposition 3.6 montre que $\Phi(u)$ est égal à $\delta(u)$, et d'après la Proposition 3.1 on a

$$
\int_{0}^{1} u_{s} d \tilde{N}_{s}= \begin{cases}\delta(u)+\sum_{j=1}^{n} \Psi_{s_{j}} u_{s_{j}}\left(s_{1}, \ldots, \hat{s}_{j}, \ldots, s_{n}\right), & \text { si } \omega=\left(s_{1}, \ldots, s_{n}\right) \\ \delta(u)+\Psi_{s_{1}} u_{s_{1}}(e), & \text { si } \omega=s_{1} \\ \delta(u), & \text { si } \omega=e .\end{cases}
$$

Si nous définissons le processus

$$
A_{s}(\omega) \equiv \begin{cases}u_{s}(\omega)-u_{s}(\omega-\{s\}), & \text { si } s \in \omega=\left(s_{1}, \ldots, s_{n}\right), n>1 \\ u_{s}(s)-u_{s}(e), & \text { si } \omega=(s) \\ 0, & \text { dans les autres cas. }\end{cases}
$$

Alors

$$
\int_{0}^{1} A_{s}(\omega) d N_{s}= \begin{cases}\sum_{j=1}^{n} \Psi_{s_{j}} u_{s_{j}}\left(s_{1}, \ldots, \hat{s}_{j}, \ldots, s_{n}\right) \\ =\sum_{j=1}^{n} A_{s_{j}}, & \text { si } \omega=\left(s_{1}, \ldots, s_{n}\right), n>1 \\ \Psi_{s_{1}} u_{s_{1}}(e), & \text { si } \omega=s_{1} \\ 0, & \text { dans les autres cas }\end{cases}
$$

et la proposition suivante est alors immédiate:

Proposition 3.7. Soit $u=\left\{u_{t}, t \in[0,1]\right\}$ un processus qui satisfait les conditions de la Proposition 3.1. Supposons que $u \in L^{2}(\Omega \times[0,1])$ et que $\Phi(u) \in L^{2}(\Omega)$. Alors il existe un processus $\left\{A_{t}, t \in[0,1]\right\}$ tel que

$$
\delta(u)=\int_{0}^{1} u_{s} d \tilde{N}_{s}-\int_{0}^{1} A_{s} d N_{s}, \quad \omega-p . s .
$$




\section{Les intégrales rétrograde et progressive et l'intégrale trajectorielle}

Dans cette section nous arriverons à la relation donnée dans la Proposition 3.1 à partir des définitions de l'intégrale progressive et rétrograde d'un bon processus $f$, ce qui présente un point de vue différent et peutêtre plus naturel.

Soient $X$ et $Y$ deux processus indexés par $[0,1]$ tels que leurs trajectoires et celles de $X Y$ appartiennent à $L^{1}([0,1])$ p.s. Alors Russo et Vallois $[\mathbf{R u}-\mathbf{V a - 1}]$, définissent les intégrales progressive et rétrograde respectivement comme les limites en probabilité suivantes

$$
\begin{aligned}
& \lim _{n \rightarrow \infty} n \int_{0}^{1} X_{t}\left(Y_{\left(t+\frac{1}{n}\right) \wedge 1}-Y_{t}\right) d t, \\
& \lim _{n \rightarrow \infty} n \int_{0}^{1} X_{t}\left(Y_{t}-Y_{\left(t-\frac{1}{n}\right) \vee 0}\right) d t,
\end{aligned}
$$

lorsqu'elles existent.

Si $Y$ est le processus de Poisson et le processus $X$ a trajectoires càdlàg et si on définit les intégrales par des limites presque sûres, c'est une conséquence d'un lemme de Russo et Vallois [Ru-Va-2] que l'intégrale progressive coincide avec l'intégrale trajectorielle de $X_{s-}$ par rapport au processus de Poisson compensé. Dans la proposition suivante nous présentons une preuve directe de ce résultat.

Proposition 4.1. Soit $f=\left\{f_{t}, t \in[0,1]\right\}$ un processus mesurable à trajectoires càdlàg. Alors

$$
\lim _{n \rightarrow \infty} n \int_{0}^{1} f_{t}\left(\tilde{N}_{\left(t+\frac{1}{n}\right) \wedge 1}-\tilde{N}_{t}\right) d t=\int_{0}^{1} f_{t-} d \tilde{N}_{t}, \quad \omega-p . s .
$$

Preuve: Pour $\omega=\left\{s_{1}, s_{2}, \ldots, s_{n}\right\}, 0<s_{1}<s_{2}<\cdots<s_{n}$ fixé, nous considèrons l'intégrale

$$
n \int_{0}^{1} f_{t}(\omega)\left(\tilde{N}_{\left(t+\frac{1}{n}\right) \wedge 1}-\tilde{N}_{t}\right) d t=n \int_{0}^{1} f_{t}(\omega)\left(\int_{t}^{\left(t+\frac{1}{n}\right) \wedge 1} d \tilde{N}_{s}\right) d t
$$


que l'on peut ecrire par application du lemme de Fubini

$$
\begin{aligned}
& n \int_{0}^{\frac{1}{n}}\left[\int_{0}^{s} f_{t}(\omega) d t\right] d \tilde{N}_{s}+n \int_{\frac{1}{n}}^{1}\left[\int_{s-\frac{1}{n}}^{s} f_{t}(\omega) d t\right] d \tilde{N}_{s} \\
= & n \int_{0}^{\frac{1}{n}}\left[\int_{0}^{s} f_{t}(\omega) d t\right] d N_{s}-n \int_{0}^{\frac{1}{n}}\left[\int_{0}^{s} f_{t}(\omega) d t\right] d s \\
+ & n \int_{\frac{1}{n}}^{1}\left[\int_{s-\frac{1}{n}}^{s} f_{t}(\omega) d t\right] d N_{s}-n \int_{\frac{1}{n}}^{1}\left[\int_{s-\frac{1}{n}}^{s} f_{t}(\omega) d t\right] d s .
\end{aligned}
$$

Quand $n \rightarrow \infty$ les deux premières intégrales tendent vers zéro par bornitude des trajectoires.

La troisième intégrale

$$
n \int_{\frac{1}{n}}^{1}\left[\int_{s-\frac{1}{n}}^{s} f_{t}(\omega) d t\right] d N_{s}=n \sum_{s_{i}>\frac{1}{n}} \int_{s_{i}-\frac{1}{n}}^{s_{i}} f_{t}(\omega) d t
$$

converge vers $\int_{0}^{1} f_{s-} d N_{s}$, et finalement la dernière intégrale a pour limite $\int_{0}^{1} f_{s-} d s$.

Pour $\omega=e$ le résultat est également vrai.

Remarque 4.2. Si on considère le cas rétrograde la limite est l'intégrale trajectorielle $\int_{0}^{1} f_{t} d \tilde{N}_{t}$.

Remarque 4.3. Dans le cas Poisson, pour passer de l'intégrale progressive entre 0 et $t$ à la rétrograde on doit ajouter à la première la variation quadratique $[f, N]_{t}$.

Kurtz, Pardoux et Protter [K-P-P $]$ considèrent des équations différentielles Stratonovich conduites par des semimartingales générales que ont des solutions avec des bonnes propriétés, et où l'intégrale Stratonovich n'est pas consistent avec la definition de Protter $[\mathbf{P}]$, et seulement est définie pour des integrands qui sont solutions des équations. Dans le cas Poisson cette intégrale est l'intégrale progressive plus $1 / 2[f, N]$.

Dans la proposition suivante on arrivera à la formule de la Proposition 3.1, pour des processus cadlag avec certaines hypothèses supplémentaires, mettant en évidence le passage à la limite. 
Proposition 4.4. Soit $f=\left\{f_{t}: t \in[0,1]\right\}$ un processus mesurable tel que

(i) Ses trajectoires sont cadlag.

(ii)

$$
\int_{0}^{1} \int_{0}^{1}\left|f_{t}\left(w+\delta_{s}\right)\right| d s d t<\infty
$$

(iii) Il existe un processus $Y$ à trajectoires dans $L^{1}([0,1])$ tel que pour presque tout $\omega$,

$$
n \int_{\left(s-\frac{1}{n}\right) \vee 0}^{s}\left|f_{t}\left(\omega+\delta_{s}\right)\right| d t \leq Y_{s}(\omega), \quad \forall n, \quad s \in[0,1] .
$$

Alors, $\omega$-p.s.

$$
\int_{0}^{1} f_{t-} d \tilde{N}_{t}=\Phi\left(f_{-}\right)+\Phi\left(\Psi f_{-}\right)+\int_{0}^{1} \Psi_{s} f_{s-} d s .
$$

Remarque 4.5. Le processus $f_{-}=\left\{f_{t-}: t \in[0,1]\right\}$ est prévisible si $f$ est adapté. Donc la Proposition 4.4 et la Remarque 3.3 impliquent dans ce cas que

$$
\int_{0}^{1} f_{t-} d \tilde{N}_{t}=\Phi\left(f_{-}\right)
$$

Remarque 4.6. Si dans l'hypothèse (iii) on change les limites d'intégration par $s$ et $\left(s+\frac{1}{n}\right) \wedge 1$, alors on a

$$
\begin{aligned}
\lim _{n \rightarrow \infty} n \int_{0}^{1} f_{t}\left(\tilde{N}_{t}-\tilde{N}_{\left(t-\frac{1}{n}\right) \vee 0}\right) d t & =\int_{0}^{1} f_{t} d \tilde{N}_{t} \\
& =\Phi(f)+\Phi(\Psi f)+\int_{0}^{1} \Psi_{s} f_{s} d s, \quad \omega \text {-p.s. }
\end{aligned}
$$

Preuve: Aprés la Proposition 4.1 il faut prouver que

$$
\lim _{n \rightarrow \infty} n \int_{0}^{1} f_{t}\left(\tilde{N}_{\left(t+\frac{1}{n}\right) \wedge 1}-\tilde{N}_{t}\right) d t=\Phi\left(f_{-}\right)+\Phi\left(\Psi f_{-}\right)+\int_{0}^{1} \Psi_{s} f_{s-} d s .
$$

Considérons le processus déterministe de paramètre $s$,

$$
1_{] t,\left(t+\frac{1}{n}\right) \wedge 1\right]}(s) .
$$

Par définition de l'opérateur $\Phi$ on peut écrire

$$
\tilde{N}_{\left(t+\frac{1}{n}\right) \wedge 1}-\tilde{N}_{t}=\Phi\left(1_{] t,\left(t+\frac{1}{n}\right) \wedge 1\right]}(s)\right) .
$$


Alors

$$
n \int_{0}^{1} f_{t}\left(\tilde{N}_{\left(t+\frac{1}{n}\right) \wedge 1}-\tilde{N}_{t}\right) d t=n \int_{0}^{1} f_{t} \Phi\left(1_{] t,\left(t+\frac{1}{n}\right) \wedge 1\right]}(s)\right) d t .
$$

Par l'hypothèse (ii) $\left(\Psi_{s} f_{t}\right) 1_{\left.] t,\left(t+\frac{1}{n}\right) \wedge 1\right]}(s) \in L^{1}([0,1])$, t-p.s. Donc le Lemme 2.5 implique que cette intégrale est égale à

$$
\begin{aligned}
& n \int_{0}^{1} \Phi\left(f_{t} 1_{] t,\left(t+\frac{1}{n}\right) \wedge 1\right]}(s)\right) d t \\
+ & n \int_{0}^{1} \Phi\left(\left(\Psi_{s} f_{t}\right) 1_{] t,\left(t+\frac{1}{n}\right) \wedge 1\right]}(s)\right) d t \\
+ & n \int_{0}^{1}\left[\int_{0}^{1}\left(\Psi_{s} f_{t}\right) 1_{] t,\left(t+\frac{1}{n}\right) \wedge 1\right]}(s) d s\right] d t,
\end{aligned}
$$

et notons que toutes ces intégrales existent.

Nous allons étudier chaque terme

(A1) On peut voir que nous sommes dans les conditions du Lemme 2.4 et donc on peut interchanger l'opérateur $\Phi$ avec l'intégrale dans (A1), pour obtenir

$$
n \Phi\left(\int_{0}^{1} f_{t} 1_{] t,\left(t+\frac{1}{n}\right) \wedge 1\right]}(s) d t\right)=\Phi\left(n \int_{\left(s-\frac{1}{n}\right) \vee 0}^{s} f_{t} d t\right) .
$$

Comme

$$
u_{s}^{n} \equiv n \int_{\left(s-\frac{1}{n}\right) \vee 0}^{s} f_{t} d t \longrightarrow f_{s-},
$$

ponctuellement et dans $L^{1}([0,1])$, par le Lemme 2.6 on a

$$
\Phi\left(n \int_{\left(s-\frac{1}{n}\right) \vee 0}^{s} f_{t} d t\right) \underset{n \rightarrow \infty}{\longrightarrow} \Phi\left(f_{s-}\right) .
$$

(A2) Appliquant aussi le Lemme 2.4 au terme (A2) on obtient

$$
\Phi\left(n \int_{0}^{1}\left(\Psi_{s} f_{t}\right) 1_{] t,\left(t+\frac{1}{n}\right) \wedge 1\right]}(s) d t\right)=\Phi\left(n \int_{\left(s-\frac{1}{n}\right) \vee 0}^{s}\left(\Psi_{s} f_{t}\right) d t\right) .
$$

Notons que

$$
n \int_{\left(s-\frac{1}{n}\right) \vee 0}^{s}\left(\Psi_{s} f_{t}\right) d t=n \int_{\left(s-\frac{1}{n}\right) \vee 0}^{s}\left(f_{t}\left(\omega+\delta_{s}\right)-f_{t}(\omega)\right) d t
$$


a pour limite $\Psi_{s} f_{s-}$, et l'hypothèse (iii) implique que la convergence à lieu aussi au sens $L^{1}([0,1])$. Alors par le Lemme 2.6

$$
\Phi\left(n \int_{\left(s-\frac{1}{n}\right) \vee 0}^{s}\left(\Psi_{s} f_{t}\right) d t\right) \longrightarrow \Phi\left(\Psi_{s} f_{s-}\right) .
$$

(A3) La convergence au sens $L^{1}([0,1])$ du cas précedent implique que

$$
\int_{0}^{1}\left[n \int_{\left(s-\frac{1}{n}\right) \vee 0}^{s} \Psi_{s} f_{t} d t\right] d s \longrightarrow \int_{0}^{1} \Psi_{s} f_{s-} d s .
$$

\section{Références}

[B] P. Biane, Calcul stochastique non-commutatif, in "Lectures on Probability Theory (Saint-Flour, 1993)", Lecture Notes in Mathematics 1608, Springer, Berlin, 1995, pp. 1-96.

[K-P-P] T. G. Kurtz, E. Pardoux et P. Protter, Stratonovich stochastic differential equations driven by general semimartingales, Ann. Inst. H. Poincaré Probab. Statist. 31(2) (1995), 351-377.

[L-R-T] J. A. León, J. Ruiz de Chávez et C. Tudor, On equivalence of solutions to anticipating semilinear stochastic equations on the Poisson space, Rev. Roumaine Math. Pures Appl. 41(9-10) (1996), 649-661.

[Ne] J. Neveu, Processus ponctuels, in "École d'Été de Probabilités de Saint-Flour, VI-1976", Lecture Notes in Mathematics 598, Springer-Verlag, Berlin, 1977, pp. 249-445.

[N-V-1] D. Nualart et J. Vives, Anticipative Calculus for the Poisson process based on the Fock space, in "Séminaire de Probabilités XXIV, 1988/89", Lecture Notes in Mathematics 1426, Springer, Berlin, 1990, pp. 154-165.

[N-V-2] D. Nualart et J. Vives, A duality formula on the Poisson space and some applications, in "Seminar on Stochastic Analysis, Random Fields and Applications (Ascona, 1993)", Progr. Probab. 36, Birkhäuser, Basel, 1995, pp. 205-213.

[P] P. ProtTer, "Stochastic integration and differential equations. A new approach", Springer-Verlag, Berlin, 1990.

[P-S-V] N. Privault, J. L. Solé et J. Vives, Chaotic Kabanov formula for the Azéma martingales, Bernoulli (to appear).

[Ru-Va-1] F. Russo ET P. VALLOIs, Intégrales progressive, rétrograde et symétrique de processus non-adaptés, C. R. Acad. Sci. Paris Sér. I Math. 312(8) (1991), 615-618. 
[Ru-Va-2] F. Russo et P. Vallois, The generalized covariation process and Itô formula, Stochastic Process. Appl. 59(1) (1995), 81-104.

Jorge A. León:

CINVESTAV-IPN

Departamento de Matemáticas

Apartado postal 14-740

07000 México, D.F.

México

E-mail address: jleon@math.cinvestav.mx

Josep L. Solé et Josep Vives:

Departament de Matemàtiques

Universitat Autònoma de Barcelona

08193 Bellaterra (Barcelona)

Spain

E-mail address: jllsole@mat.uab.es

E-mail address: vives@mat.uab.es

Primera versió rebuda el 18 de maig de 1999, darrera versió rebuda el 28 d'octubre de 1999. 